ACCEPTED MANUSCRIPT

\title{
Effect of subsurface voids on the nanoindentation of Fe crystals
}

To cite this article before publication: Juan A Hofer et al 2020 Modelling Simul. Mater. Sci. Eng. in press https://doi.org/10.1088/1361$651 \mathrm{X} / \mathrm{ab} 6915$

\section{Manuscript version: Accepted Manuscript}

Accepted Manuscript is "the version of the article accepted for publication including all changes made as a result of the peer review process, and which may also include the addition to the article by IOP Publishing of a header, an article ID, a cover sheet and/or an 'Accepted Manuscript' watermark, but excluding any other editing, typesetting or other changes made by IOP Publishing and/or its licensors"

This Accepted Manuscript is ( 2020 IOP Publishing Ltd.

During the embargo period (the 12 month period from the publication of the Version of Record of this article), the Accepted Manuscript is fully protected by copyright and cannot be reused or reposted elsewhere.

As the Version of Record of this article is going to be / has been published on a subscription basis, this Accepted Manuscript is available for reuse under a CC BY-NC-ND 3.0 licence after the 12 month embargo period.

After the embargo period, everyone is permitted to use copy and redistribute this article for non-commercial purposes only, provided that they adhere to all the terms of the licence https://creativecommons.org/licences/by-nc-nd/3.0

Although reasonable endeavours have been taken to obtain all necessary permissions from third parties to include their copyrighted content within this article, their full citation and copyright line may not be present in this Accepted Manuscript version. Before using any content from this article, please refer to the Version of Record on IOPscience once published for full citation and copyright details, as permissions will likely be required. All third party content is fully copyright protected, unless specifically stated otherwise in the figure caption in the Version of Record.

View the article online for updates and enhancements. 


\title{
Effect of subsurface voids on the nanoindentation of Fe crystals
}

\author{
Juan A. Hofer, ${ }^{1}$ Carlos J. Ruestes, $,{ }^{2},{ }^{*}$ Eduardo M. Bringa, ${ }^{3}$ and Herbert M. Urbassek ${ }^{4, \dagger}$ \\ ${ }^{1}$ Instituto Balseiro, Universidad Nacional de Cuyo and Comisión Nacional de Energía Atómica, \\ Av. Bustillo 9500, (8400) San Carlos de Bariloche, Argentina \\ ${ }^{2}$ Instituto Interdisciplinario de Ciencias Básicas, Universidad Nacional de Cuyo, \\ CONICET, Facultad de Ciencias Exactas y Naturales, \\ Padre J. Contreras 1300, (5500) Mendoza, Argentina. \\ ${ }^{3}$ CONICET, Facultad de Ingeniería, Universidad de Mendoza, \\ (5500) Mendoza, Argentina, and Centro de Nanotecnología Aplicada, \\ Facultad de Ciencias, Universidad Mayor, Chile. \\ ${ }^{4}$ Physics Department and Research Center OPTIMAS, University Kaiserslautern, \\ Erwin-Schrödinger-Straße, D-67663 Kaiserslautern, Germany
} (Dated: December 13, 2019)

\begin{abstract}
Subsurface voids may strongly affect the response of materials to nanoindentation. We explore these effects for a bcc single-crystalline Fe sample using molecular dynamics simulation. Deformation occurs mainly by nucleation and propagation of dislocations. As dislocations impinge into the voids, these suffer a reduction in volume, consistent with mass transfer mechanisms. Our results show that voids act as highly efficient absorbers of dislocations, effectively limiting the extension of the plastic zone. Surprisingly, mechanical properties are marginally affected by the presence of voids in the range of sizes and spatial distributions tested, except for voids a few nanometers below the surface. Deformation twinning is observed as a transient effect in some cases; however, for voids close enough to the indentation area, no twinning was found.

Keywords: molecular dynamics, nanoindentation, dislocations, voids, plasticity
\end{abstract}

\section{INTRODUCTION}

Nanoindentation is an extremely versatile technique to probe a variety of materials and properties [1-4]. It is commonly used to gather information about the elastic modulus and hardness of a material. It can also be used to assess fracture toughness, adhesion, strain hardening, etc. Atomistic studies based on molecular dynamics (MD) simulations have provided valuable understanding of the deformation mechanisms (such as the generation, movement and reactions of dislocations) taking place during nanoindentation [5-8].

The vast majority of simulations consider the substrate to be a defect-free, ideal crystal. The justification lies in the small size of simulation volumes in MD, of the order of several tens of nm. For defects such as point defects, dislocations or grain boundaries, and assuming typical experimental concentrations, none or only a few lattice imperfections will lie in the simulation volumes.

In order to study the effect of imperfections on the indentation behavior, several dedicated studies have included lattice defects in the simulation volume by purpose. The inclusion of grain boundaries [9-11] had shown that they may act as a sink for dislocations, or even reflect or emit dislocations. Further work along these lines has been reviewed in [8]. The effect of dislocations on fcc materials was studied by Ukwatta and Achuthan [12], by

* Electronic address: cruestes@fcen.uncu.edu.ar ${ }^{\dagger}$ Electronic address: urbassek@rhrk.uni-kl.de; URL: http:// www. physik.uni-kl.de/urbassek/ introducing a small number (up to 3 ) of dislocations into the sample and found that dislocation nucleation is alleviated by their presence. The effect of a high statistically stored dislocation density on bcc metals was studied by Alhafez et al. [13] and found that the dislocation-filled crystal exhibits a strongly shortened elastic regime compared to a defect-free one. The effect of small bumps on the surface - which may exist in the form of adatom islands - on the indentation process was studied in [14]. There it was found that the maximum effect on indentation occurs when the bump size is of the order of the contact radius; then the surface is weakest and yields earliest. As another important type of defects, the effect of voids in the simulation volume has been studied. Voids can act as stress concentrators, assisting the nucleation of dislocations [15]; in addition, due to their internal surfaces, they may also annihilate dislocations. Atomistic simulations had been used to study the effects of vacancies on the nanoindentation response of fcc and bcc metals [16-19], showing that the presence of vacancies can significantly lower the yield load. Zhao et al. [20] used atomistic simulations to explore the nanoindentation response of fcc $\mathrm{Cu}$ with a void under the surface, concluding that the position and size of the void have an influence on the plastic deformation process. A similar conclusion was reached by Abu-Shams and Shabib [21] when studying a bcc Fe- $10 \% \mathrm{Cr}$ alloy, and by Shan et al. [22] and Tan and Jeng [23] in their studies on $\mathrm{Cu}$.

Our motivation for this study lies on the fact that voids are one of the prototypical defects that can be found in metals. Voids can also be formed by the Kirkendall effect, such as in integrated circuits and electronics devices $[24,25]$. Last but not least, voids can be formed due to 
imperfections of the technological processes involved on metal production (sintering, powder consolidation, etc), as well as electromigration [26].

In this paper we explore how voids influence the mechanical behavior of Fe under nanoindentation. To this end we compare several simulations, one for an ideal crystal and others for crystals containing a void in different positions and sizes. The potential effect of an arrangement of voids is also explored.

\section{METHOD}

We employ molecular-dynamics simulation to study the behavior of an iron single crystal containing a void during nanoindentation.

Fig. 1 shows a schematic representation of the MD simulation system. It illustrates the configuration of the tip and the substrate. The simulation consists of three stages: (i) penetration, during which the indenter is pushed perpendicular into the substrate to a given depth; (ii) holding, during which the tip is held at the maximum penetration for a time of about one tenth of the time required for penetration; and (iii) retraction of the indenter: the indenter is moved out of the substrate to return to the initial height.

Bcc iron single crystal substrates were tested in three principal crystallographic orientations, namely (100), (110), (111). The Fe substrates have dimensions of 30 $\times 30 \times 15 \mathrm{~nm}^{3}$, for a total of $\approx 1.3$ million atoms. The proximity of the simulation boundaries may affect MD simulation results [27]. In order to prevent such effects, we checked that the box is large enough to avoid the generated plasticity (dislocations) to reach the boundaries of the simulation box. The Fe-Fe interaction is described by the Mendelev potential [28]. Periodic boundary conditions were applied in the $\mathrm{x}$ and $\mathrm{y}$ directions. In order to suppress any rigid-body movement of the substrate, two atom layers at the bottom and at the side faces of the substrate are fixed. The next four layers at the bottom and the sides are kept at a constant temperature of 300 $\mathrm{K}$ by means of a Langevin thermostat.

A void was inserted in the substrate by removing atoms within a sphere of radius $r$. Four different void radii were tested, namely $2.5 \AA, 5 \AA, 12.5 \AA$ and $25 \AA$. In each case, the amount of atoms removed on each void corresponds to 5, 55, 692 and 5538, respectively. In all cases, the indenter was positioned directly above the center of the void, with no lateral offset, see Fig. 1. We denote the distance between the upper apex of the void and the surface $d$, which is varied between 14 and $52 \AA$. For brevity we will denote $d$ as the 'wall thickness' in the following.

In addition, in Sect. IV, we study the effect of an array of voids on the indentation.

Before performing the simulations, the substrates are relaxed until all stress components have reached values $<10^{-5} \mathrm{GPa}$ [29]. The indenter tip penetration rate is chosen as $20 \mathrm{~m} / \mathrm{s}$, both for indentation and retraction.
This velocity, though high compared to experimental indentation, is well below the longitudinal wave velocity of iron, and should be considered low enough for typical MD studies.

The tip has a spherical shape with a radius of $R=$ $5.0 \mathrm{~nm}$. It interacts in a purely repulsive way with the substrate atoms according to the law [30]

$$
V(r)= \begin{cases}k(R-r)^{3}, & r<R, \\ 0, & r \geq R .\end{cases}
$$

Here $r$ is the distance of a substrate atom to the center of the indenter, and $k=10 \mathrm{eV} / \AA^{3}[30,31]$ is a constant. For the indenter model chosen, there are no tangential forces, akin to a Hertzian indenter, Maximum penetration was prescribed at $1.6 \mathrm{~nm}$.

The MD simulations are performed using the opensource LAMMPS code [32] with a constant time step of $1 \mathrm{fs}$. Visualization is performed using OVITO [33]; DXA [34] and CAT [35-37] are employed for analysis of the plasticity generated.

\section{RESULTS}

\section{A. Forces}

The determination of the forces acting on the tip during nanoindentation is straightforward in an MD simulation. A normal force is required to keep the tip at its prescribed depth, $d$. Fig. 2 displays the evolution of the normal forces for tests on the three crystallographic orientations for a void of $25 \AA$ and varying depth. In general, as the void gets closer to the surface, the load required for the onset of plasticity decreases. This effect is more noticeable as the thickness of material between the void and the substrate surface is below $30 \AA$. Below such thickness, the elastic response of the substrate gets also affected, showing a reduction in stiffness, particularly for a thickness of $14 \AA$. These features hold regardless of the crystallographic orientation of the substrate.

Fig. 3 provides further insight into the effect of pore depth for a variety of pore radii on a (100) oriented single crystal. In general, as the ratio of the void radius $r$ to the wall thickness $d$ takes values of 0.1 and above, the load required for the onset of plasticity decreases as this ratio increases (wall thickness decreases). As the thickness $d$ decreases to the point that it is lower than the radius of the void, the elastic limit not only decreases but also the elastic response gets more compliant. If one can assume, as a first approximation, that the Hertzian law, $F(h)=$ $\frac{4}{3} E \sqrt{R} h^{3 / 2}$, holds for all the geometries tested here, we can see that the presence of a void close to the surface produces a decrease of $12 \%$ in the elastic modulus of the sample compared to the defect-free sample, for $d=27$ $\AA$. This difference increases as the thickness $d$ tends to smaller values ( $35 \%$ decrease for $d=14 \AA$ ). These results suggest that experimental nanoindentation of atomically 
flat surfaces might be able to determine the presence of subsurface voids due to changes in the elastic portion of the loading curves, if the subsurface void density is large and/or if void sizes are comparable to the characteristic indenter size.

The spherical indenter is expected to exert a stress field on the sample of the form of that of Hertzian solution [1]. When the void is relatively far from the area of influence of the indenter, changes are hardly seen on the loading curves. However, as the void gets closer to the surface, it imposes an iso-stress surface of zero normal stress in its radius, this strongly differs from the Hertzian solution, and it modifies the apparent mean pressure required for the onset of plasticity. The presence of a void can also alter the hardness of the sample. Following the methods presented elsewhere $[31,38]$, we found that for the [100] oriented crystal, the hardness changes from approximately $20 \mathrm{GPa}$ for the defect-free crystal to $17.5 \mathrm{GPa}$ for $d=27 \AA(r=25 \AA)$ and to $16.0 \mathrm{GPa}$ for $d=14 \AA$ and same the void radius.

After penetration and before retraction, the indenter is held in position at maximum penetration. During this holding stage, the load decreases around $15 \%-20 \%$ depending on the case. This phenomenon is well known $[5,6]$ and is caused by the relaxation of the dislocation network created by the indentation process. As the indenter is held in position, dislocations continue moving outwards in the high-stress field of the indenter tip and react, allowing the substrate stresses to relax and resulting in a decrease of the load registered.

\section{B. Deformation mechanisms}

Like in the typical response of bcc substrates [7], we observe the generation of dislocations with two different kinds of Burgers vectors, $\boldsymbol{b}=1 / 2\langle 111\rangle$ and $\boldsymbol{b}=\langle 100\rangle$; these dislocations are plotted distinctly in Fig. 4 for [100] oriented crystals, with green for the former and magenta for the latter. Although dislocations with the first Burgers vector have lower energy, $\boldsymbol{b}=\langle 100\rangle$ dislocations may form during deformation by the interaction between dislocations with Burgers vector $\boldsymbol{b}=1 / 2\langle 111\rangle$ [38-40]. This can be noticed on Fig. 4.a specially, where two $\boldsymbol{b}=1 / 2\langle 111\rangle$ dislocations are reacting to form a $\boldsymbol{b}=\langle 100\rangle$.

Dislocations nucleate homogeneously in the region between the void surface and the substrate surface. Previous studies on dislocation nucleation from near surface void under static tensile stress had revealed that stresses can promote the nucleation of dislocations on the void surface [41]. In our studies, no heterogeneous nucleation was found in the simulations presented here, neither at the void surface not at the substrate surface. This can be rationalized based on the findings of Tang et al. [42]; as the void radius decreases, the stress required for dislocation nucleation at its surface increases. A quick extrapolation suggests that for void radii of $25 \AA$ and below, one would expect a shear stress for dislocation nucleation of $20 \mathrm{GPa}$ and above, a stress level not achieved in our simulations.

As dislocations nucleate and propagate, they impinge on the void, producing a reduction in its volume. As a quantitative example, for the [111]-oriented crystal with a void of $25 \AA$ and $d=37 \AA$, the void suffered a decrease in volume of $1.1 \%$ during the elastic regime and as the first dislocation impinged on its surface, the void suffered a sudden additional decrease in volume of $1.3 \%$. Subsequent arrival of dislocations into the void produce additional reduction in volume. For details on dislocationmediated mass transport into voids, see $[43-45]$. Zhao et al. [20] also reported on partial void collapse during nanoindentation of $\mathrm{Cu}$ single crystals. They attribute this effect to plastic deformation processes, without explicitly mentioning mass transport by dislocations. However, their atomistic images seem to suggest that voids reduce their volume due to impingement of dislocations, as we report here.

Upon retraction, in most cases a few dislocations remain attached to the void surfaces. For the case in which the void is at its shallowest depth in the [100] oriented substrate, no dislocations were found upon retraction of the indenter (See Fig. 3.c); a few vacancies were detected, resulting from dislocation interactions.

In the experimental work of Bahr et al. [46], the authors discussed the possibility that dislocations generated during indentation might retreat during unloading. More recently, Montagne et al. [47] performed an experimental study of indentation into $\mathrm{MgO}$, providing an analysis that supports the assumption of dislocation annihilation during unloading.

Similar scenarios were found for indentations on the other crystallographic orientations, Fig. 5.

The evolution of the total length of dislocations was calculated by DXA and the results are presented on Fig. 6. The plots provide a quantitative representation of the phenomena explained in the preceding paragraphs. Upon nucleation, dislocation length increases, reaching its maximum at maximum penetration. During the holding stage, at which the indenter is kept at constant depth, dislocation length decreases by small amount, in general. Upon retraction of the indenter, the total dislocation length decreases by $1 / 3-1 / 2$ of its peak value, except for a few cases, as explained above, where complete dislocation annihilation was found and, therefore, the total dislocation length goes to zero. The reduction in total dislocation length is understandable as the stress release after retraction allows the crystal to lower its potential energy by annihilating defects.

The extension of the plastic zone was computed following the criteria presented elsewhere [48], and the dislocation densities after indent and after retraction are assembled in Table I. Clearly the total length of the dislocation network decreases during indenter removal and also the size of the plastic zone shrinks. This is in agreement with previous findings [48] and is caused by the inward 
motion of the dislocations that lead to dislocation reactions or even annihilation, while the stress field exerted by the indenter vanishes. The change of the dislocation density during indenter retraction is less predictable as it is given by the ratio of two decreasing quantities (dislocation length and plastic-zone volume).

Note the high dislocation density for $d=27 \AA$, obtained after the concentration of a high number of dislocations (Fig. 4.b) in a significantly reduced plastic zone. Also for the case of $d=14 \AA$, the total dislocation length is zero after retraction and the plastic zone size is hence null; this is in agreement with Fig. 4.c, as all the dislocations vanished after retraction.

For our simulations on defect-free crystals, twinning was found, in agreement with previous MD nanoindentation studies on iron [38]. Twinning was also found on the simulations for $d=37 \AA$ and above, albeit as a transient state, as no twinning was found after unloading. An example is presented in Fig. 7 for the case of $d=$ $52 \AA$, where it can be seen that the planar arrangement of green atoms marking the twin boundaries at full penetration does not survive after unloading. No twinning was found for wall thicknesses below $37 \AA$, presumably because the presence of a void close to the nucleation region alleviates stress, so that the threshold for twinning nucleation is not reached.

Iron is a magnetic material and high pressures sustained during sufficient time would lead to solid-solid phase changes. However, at relatively high strain rates as the ones used in nanoindentation MD simulations, tranisformation kinetics require pressures near $20 \mathrm{GPa}$ [49], much higher than thermodynamic value of $13 \mathrm{GPa}$ of the $\alpha-\epsilon$ phase change. Pressures in our MD simulations were found not large enough to drive this transformation.

In order to explore the effect of the indenter radius on the response of substrates with a void, we also tested a [100] oriented sample with a $25 \AA$ void at depth $d=14$ $\AA$ under the influence of a tip with $2.5 \mathrm{~nm}$ radius. For a penetration of $16 \AA$, we found that a smaller radius produces a total dislocation length of $151 \AA$ compared to the $345 \AA$ length produced by the $5 \mathrm{~nm}$ tip and that these dislocations are confined to a rather smaller plastic zone, see Fig. 8. We conclude that for smaller tips, the efficiency of the void to arrest dislocations is enhanced. It is expect that if the indenter radius gets large enough, dislocations would easily pass around the void and further propagate into the substrate, reducing the efficiency of the void as a dislocation arrester.

\section{AN ARRAY OF VOIDS}

The preceding sections seem to suggest that a void under the indented region can mitigate the propagation of dislocations, effectively limiting the plastic zone. To gain more insights into this possibility, we explore a more complex and realistic scenario of a substrate containing several voids. Fe single crystals with an array of four voids were indented. (100) oriented single crystals were chosen for this example, based on the fact that in such orientation, four equally oriented $\langle 111\rangle$ slip directions would penetrate into the sample. Each of the four voids were specifically located with its center along the $\langle 111\rangle$ direction formed by the point of contact of indenter and the void center. Each void had a radius of $25 \AA$ and several depths of the array were explored, namely 75,27 and $14 \AA$. In these examples, a larger substrate of $40 \times 40$ x $20 \mathrm{~nm}^{3}$ was built (2.7 million atoms). The simulation configuration is depicted in Fig. 9.

Fig. 10a displays the evolution of the normal forces for void arrays with varying depth. For a depth as low as $27 \AA$, changes are almost negligible for the elastic regime and so is the load for the onset of plasticity. As the depth of the array is reduced to $14 \AA$, the elastic response gets more compliant and the onset of plasticity occurs at a lower load. Inspection of the defective structures do show an effect on the deformation mechanisms for depths as large as $75 \AA$. As dislocations impinge on the void surface, they get "trapped" restricting their propagation and preventing the formation of prismatic loops via the "lasso-mechanism" [5], in contrast to defect-free crystals. As the depth of the voids gets as low as $14 \AA$, plasticity becomes highly confined by the voids, which see their empty volume reduced due to partial collapse as dislocations transport matter into the voids, as described in the previous section. The evolution of total dislocation length for each case is presented in Fig. 11 where we can see that the total dislocation length decreases by a factor of 5 for a void at its shallowest depth with respect to a void far away from the surface.

A similar dislocation confinement effect was reported by Shams and Shabib [21] when studying a Fe- $10 \% \mathrm{Cr}$ alloy with voids, and can also be seen in atomistic images of Esqué-de los Ojos [15] in their studies on the influence of pore size on the indentation behavior of $\mathrm{Cu}$ single crystals with an arrangement of voids.

Despite its limitations, this example shows that voids could be used as highly effective dislocation arresters at a marginal loss of mechanical properties.

\section{CONCLUSIONS}

Using classical molecular-dynamics simulation we study nanoindentation of bcc single crystals containing a single void and an array of them. The effect of crystal structure, surface orientation, void depth and radius were inspected to determine their influence on the mechanical response.

1. Mechanical properties are little affected by the presence of voids in the range of sizes and distribution tested here, except for voids very close to the surface $(d \leq 27 \AA)$ where the elastic response and the hardness can be significantly modified. 
2. The presence of voids limits the extension of the plastic zone.

3. Dislocations impinge into the voids, reducing their volume in a partial collapse. Dislocations transfer mass to the voids, reducing their volume suddenly as they impinge on the void surface. In general, dislocations remain attached to the voids.

4. Immediately after indentation the total dislocation length is larger than after indenter retraction; this is a consequence of relaxation processes during unloading.

5. Twinning is suppresed provided voids are close enough to the indentation zone.

6. Our results show that voids act as highly effective absorbers of dislocations.

The effects described in this paper might be experimentally observed in a variety of applications. An exam- ple are integrated circuits and electronics devices where voids can form by Kirkendall effect [24, 25] and electromigration [26]. Another example are irradiation samples, where subsurface void arrays of certain size and density might occur, helium bubbles are such a case [50, 51].

\section{Acknowledgments}

HMU acknowledges support by the Deutsche Forschungsgemeinschaft (DFG, German Research Foundation) - project number 172116086 - SFB 926. CJR and JAH acknowledge support by ANPCyT PICT-2015-0342, SiiP-UNCuyo-2019-M088, a donation by the Nvidia Corporation, and computational resources at TOKO-FCEN-UNCuyo cluster. EMB thanks funding from SIIP-UNCuyo-2019-2021 grant.
[1] K. L. Johnson, Contact mechanics (Cambridge University Press, Cambridge, 1985).

[2] A. C. Fischer-Cripps, Nanoindentation (Springer, New York, 2004), 2nd ed.

[3] R. W. Armstrong, W. L. Elban, and S. M. Walley, Int. J. Mod. Phys. B 27, 1330004 (2013).

[4] R. W. Armstrong, S. M. Walley, and W. L. Elban, Crystals 7, 21 (2017).

[5] T. P. Remington, C. J. Ruestes, E. M. Bringa, B. A. Remington, C. H. Lu, B. Kad, and M. A. Meyers, Acta Mater. 78, 378 (2014).

[6] C. J. Ruestes, A. Stukowski, Y. Tang, D. R/Tramontina, P. Erhart, B. A. Remington, H. M. Urbassek, M. A. Meyers, and E. M. Bringa, Mat. Sci. Eng. A 613, 390 (2014).

[7] C. J. Ruestes, E. M. Bringa, Y. Gao, and H. M. Urbassek, in Applied Nanoindentation in Advanced Materials, edited by A. Tiwari and S. Natarajan (Wiley, Chichester, UK, 2017), chap. 14, pp. 313-345.

[8] C. J. Ruestes, I. Alabd Alhafez, and H. M. Urbassek, Crystals 7, 293 (2017).

[9] A. Hasnaoui, P. M. Derlet, and H. Van Swygenhoven, Acta Mater. 52, 2251 (2004).

[10] G. Z. Voyiadjis and C. Zhang, Mat. Sci. Eng. A 621, 218 (2015).

[11] G. Z. Voyiadjis and M. Yaghoobi, Computational Materials Science 117, 315 (2016).

[12] A. Ukwatta and A. Achuthan, Computational Materials Science 91, 329 (2014).

[13] I. Alabd Alhafez, C. J. Ruestes, E. M. Bringa, and H. M. Urbassek, Journal of the Mechanics and Physics of Solids 132, 103674 (2019).

[14] G. Ziegenhain and H. M. Urbassek, Phys. Rev. B 81, 155456 (2010).

[15] D. Esqué-de los Ojos, E. Pellicer, and J. Sort, Materials 9, 355 (2016).

[16] E. Njeim and D. F. Bahr, Scripta materialia 62, 598 (2010).
[17] I. Salehinia and D. F. Bahr, Scripta Materialia 66, 339 (2012).

[18] I. Salehinia, V. Perez, and D. F. Bahr, Philosophical Magazine 92, 550 (2012).

[19] I. Salehinia and D. F. Bahr, Materials Science and Engineering: A 588, 340 (2013).

[20] P. Zhao, Y. Guo, and Z. Deng, Solid State Communications 301, 113694 (2019).

[21] M. Abu-Shams and I. Shabib, Mater. Express 7, 329 (2017).

[22] D. Shan, L. Yuan, Z. Xu, and B. Guo, Journal of nanoscience and nanotechnology 9, 1234 (2009).

[23] C.-M. Tan and Y.-R. Jeng, International Journal of Solids and Structures 46, 1884 (2009).

[24] B. Selikson, Proceedings of the IEEE 57, 1594 (1969).

[25] H. J. Lee and J. Yu, Journal of Electronic Materials 37, 1102 (2008).

[26] G. Schneider, G. Denbeaux, E. Anderson, B. Bates, A. Pearson, M. Meyer, E. Zschech, D. Hambach, and E. Stach, Applied physics letters 81, 2535 (2002).

[27] M. Yaghoobi and G. Z. Voyiadjis, Computational Materials Science 95, 626 (2014).

[28] M. I. Mendelev, S. Han, D. J. Srolovitz, G. J. Ackland, D. Y. Sun, and M. Asta, Philos. Mag. 83, 3977 (2003).

[29] G. Ziegenhain, H. M. Urbassek, and A. Hartmaier, J. Appl. Phys. 107, 061807 (2010).

[30] C. L. Kelchner, S. J. Plimpton, and J. C. Hamilton, Phys. Rev. B 58, 11085 (1998).

[31] G. Ziegenhain, A. Hartmaier, and H. M. Urbassek, J. Mech. Phys. Sol. 57, 1514 (2009).

[32] S. Plimpton, J. Comput. Phys. 117, 1 (1995), http://lammps.sandia.gov/.

[33] A. Stukowski, Model. Simul. Mater. Sci. Eng. 18, 015012 (2010), http://www.ovito.org/.

[34] A. Stukowski and K. Albe, Model. Simul. Mater. Sci. Eng. 18, 085001 (2010).

[35] A. Stukowski, V. V. Bulatov, and A. Arsenlis, Model. 
Simul. Mater. Sci. Eng. 20, 085007 (2012).

[36] A. Stukowski, Model. Simul. Mater. Sci. Eng. 20, 045021 (2012).

[37] A. Stukowski and A. Arsenlis, Model. Simul. Mater. Sci. Eng. 20, 035012 (2012).

[38] Y. Gao, C. J. Ruestes, and H. M. Urbassek, Comput. Mater. Sci. 90, 232 (2014).

[39] D. A. Terentyev, Y. N. Osetsky, and D. J. Bacon, Acta Mater. 58, 2477 (2010).

[40] A. Kelly and K. M. Knowles, Crystallography and crystal defects (John Wiley, Chichester, UK, 2012), 2nd ed.

[41] A. S. Pohjonen, F. Djurabekova, K. Nordlund, A. Kuronen, and S. P. Fitzgerald, J. Appl. Phys. 110, 023509 (2011).

[42] Y. Tang, E. M. Bringa, and M. A. Meyers, Acta Materialia 60, 4856 (2012).

[43] S. Traiviratana, E. M. Bringa, D. J. Benson, and M. A. Meyers, Acta Mater. 56, 3874 (2008).

[44] C. J. Ruestes, E. M. Bringa, A. Stukowski, J. F. R. Nieva, Y. Tang, and M. A. Meyers, Computational Materials Science 88, 92 (2014).

[45] Y. Cui, Z. Chen, and Y. Ju, Materials Letters 247, 67 (2019).

[46] D. F. Bahr, D. E. Kramer, and W. W. Gerberich, Acta Mater. 46, 3605 (1998).

[47] A. Montagne, C. Tromas, V. Audurier, and J. Woirgard, J. Mater. Res. 24, 883 (2009).

[48] Y. Gao, C. J. Ruestes, D. R. Tramontina, and H. M. Urbassek, J. Mech. Phys. Sol. 75, 58 (2015).

[49] N. Gunkelmann, E. M. Bringa, D. R. Tramontina, C. J. Ruestes, M. J. Suggit, A. Higginbotham, J. S. Wark, and H. M. Urbassek, Phys. Rev. B 89, 140102 (2014).

[50] L. Yang, H. Deng, F. Gao, H. Heinisch, R. Kurtz, S. Hu, Y. Li, and X. Zu, Nuclear Instruments and Methods in Physics Research Section B: Beam Interactions with Materials and Atoms 303, 68 (2013).

[51] H. Trinkaus and B. Singh, Journal of Nuclear Materials 323, 229 (2003). 


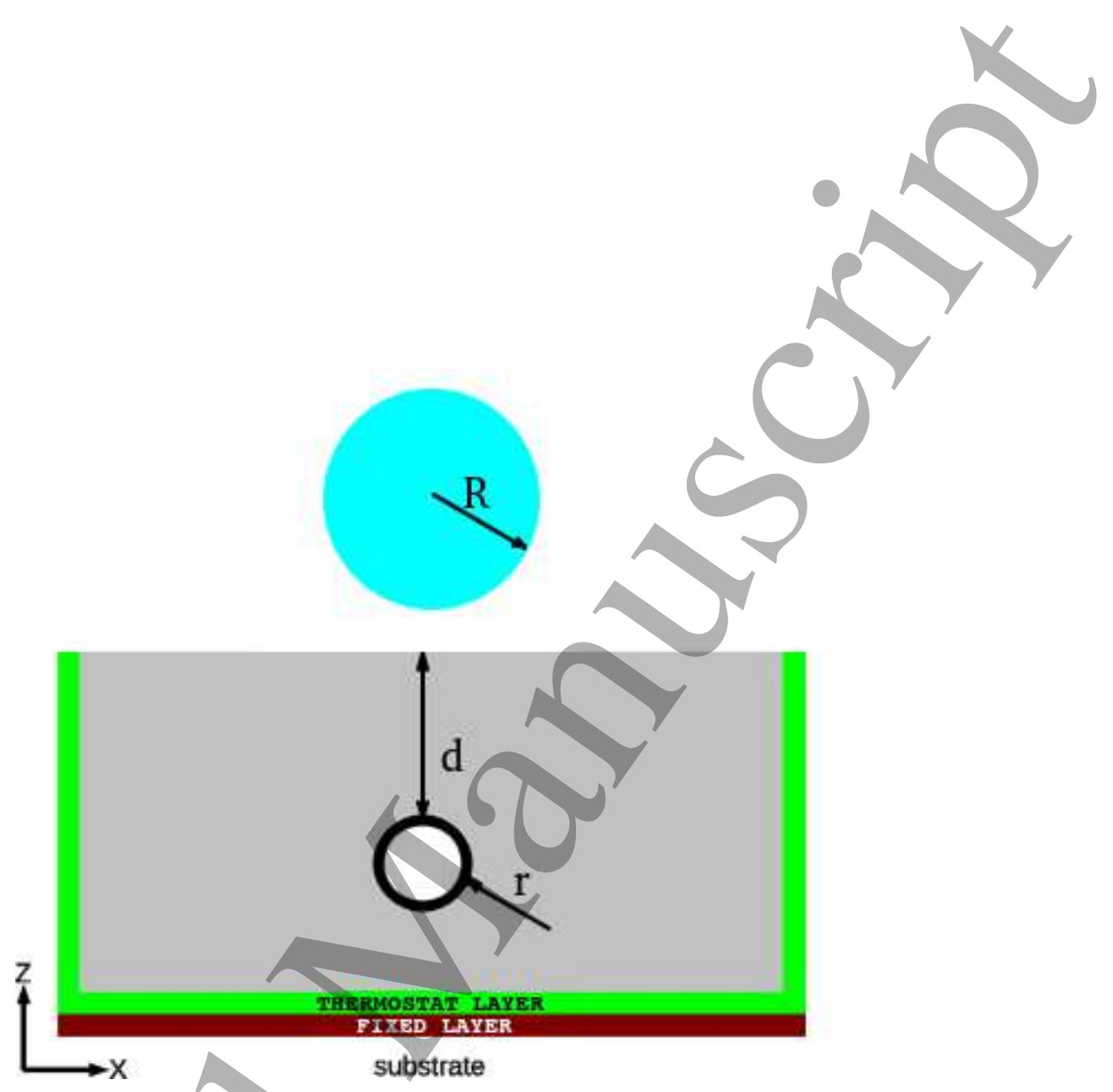

FIG. 1: Setup of the simulation system. The radius $R$ of the indenter, the radius $r$ of the void and the distance between the upper apex of the void and the surface $d$ are indicated. The substrate has thermostatting and rigid zones at its boundaries. 


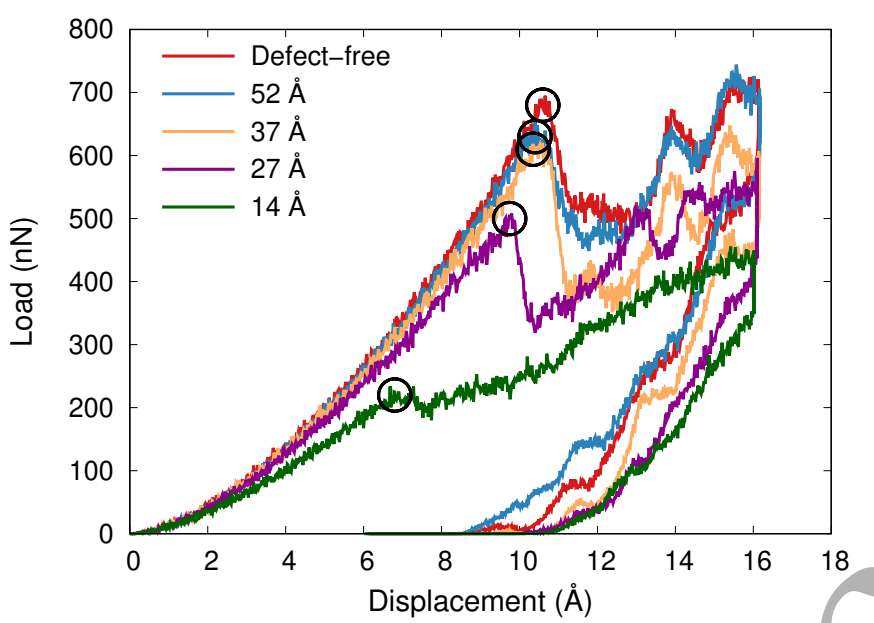

(a)
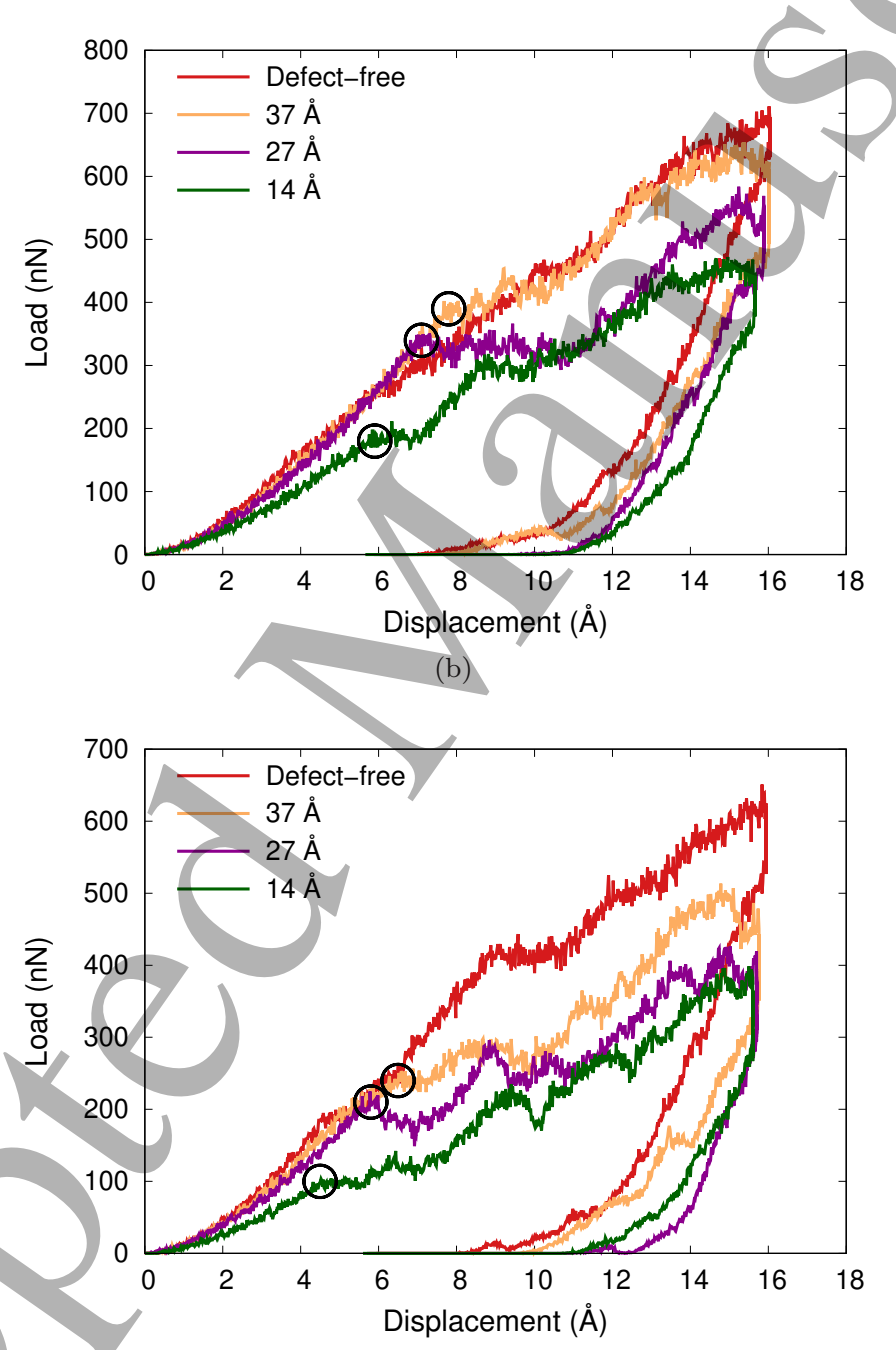

(c)

FIG. 2: Loading curves for a substrate with a void (25 A radius) at different depths. a) [100], b) [110] and c) [111]-oriented single crystals. Circles mark the onset of plasticity. 


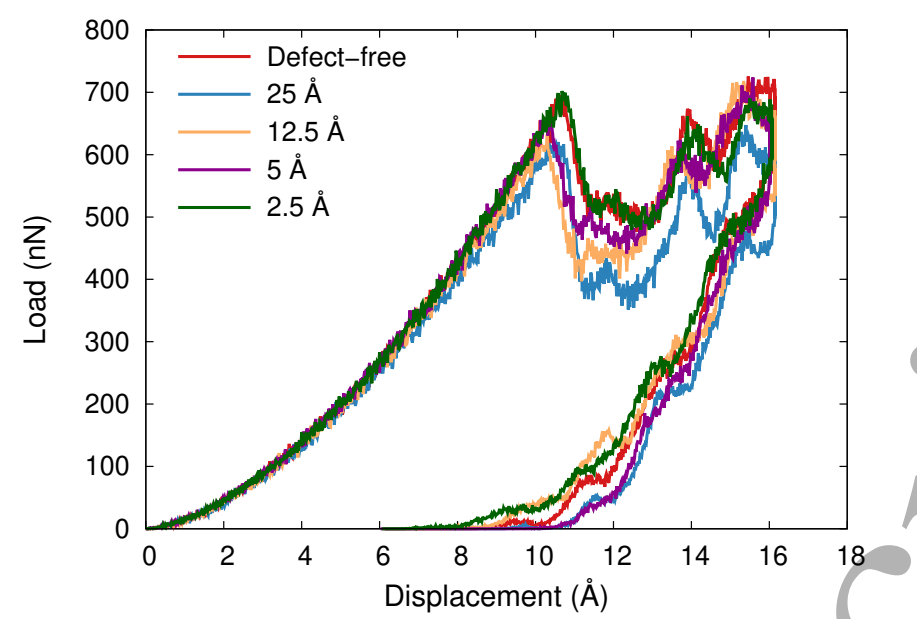

(a)
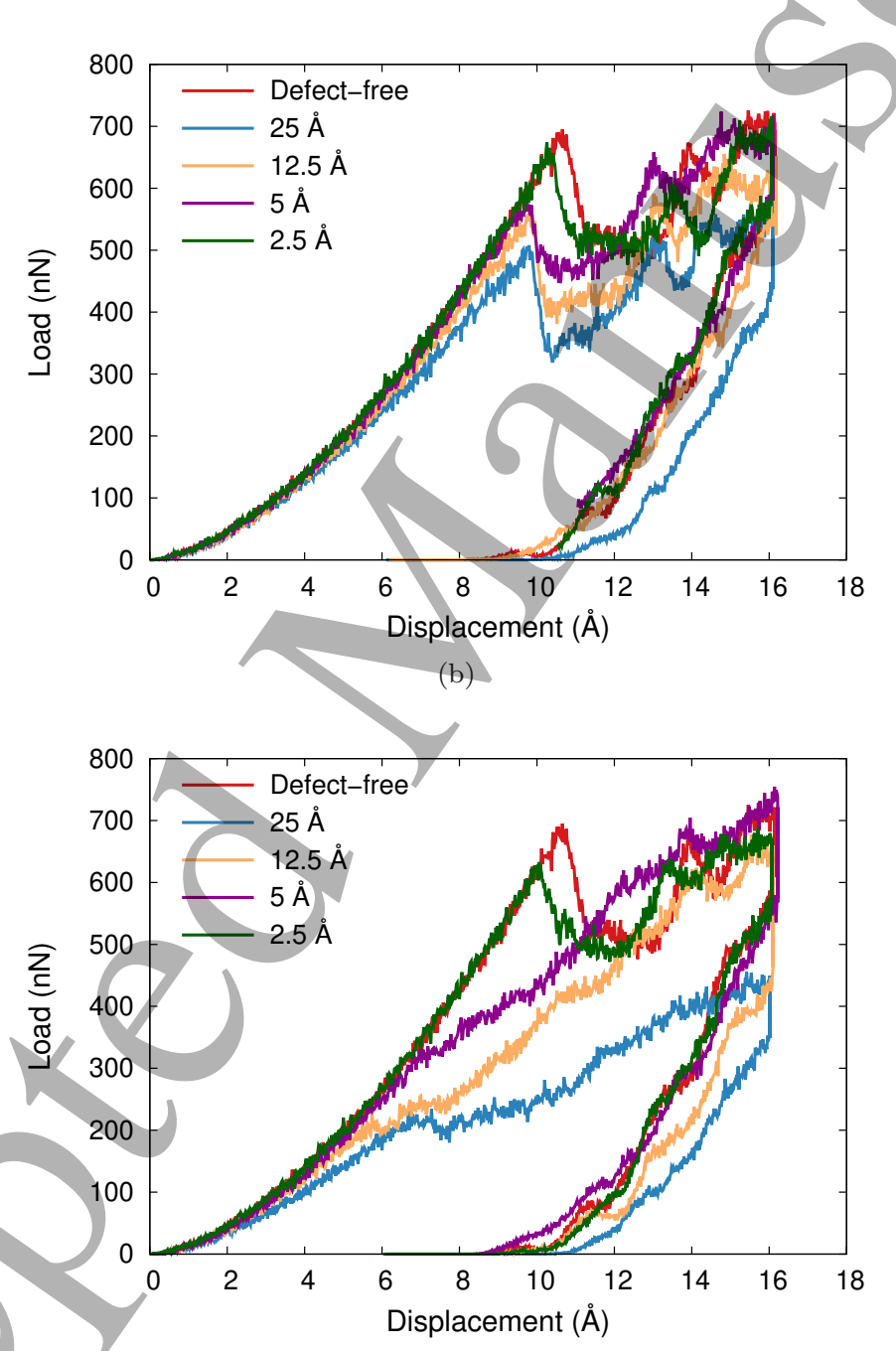

(c)

FIG. 3: Influence of the void radius for various void depths in [100] oriented single crystals. a) $d=37 \AA$, b) $27 \AA$ and c) $14 \AA$. 


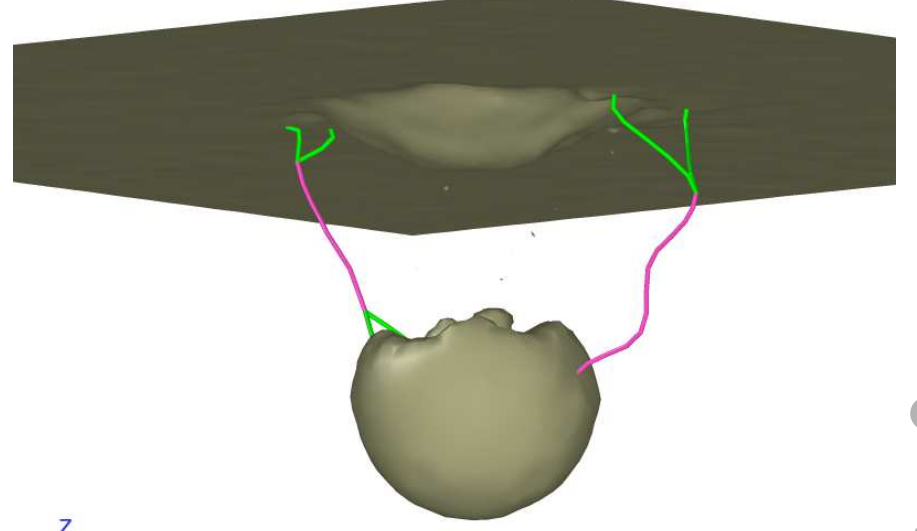

(a)

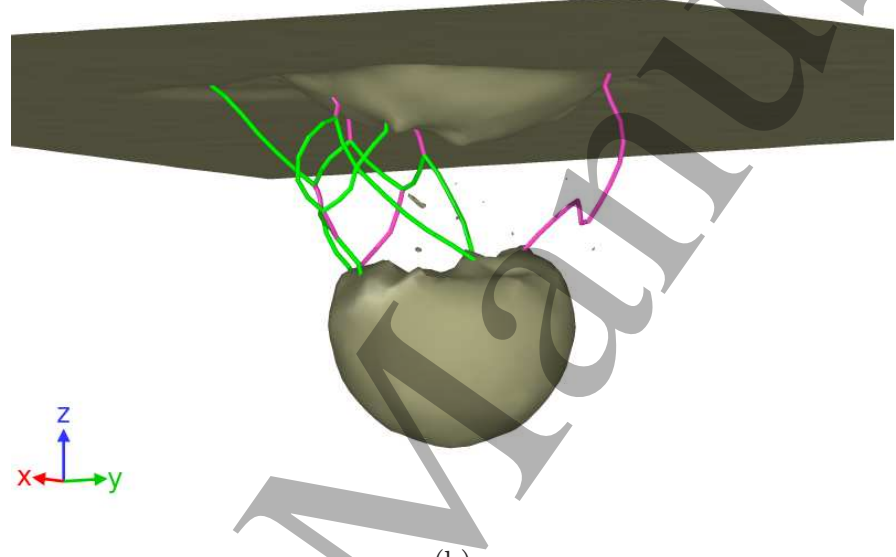

(b)
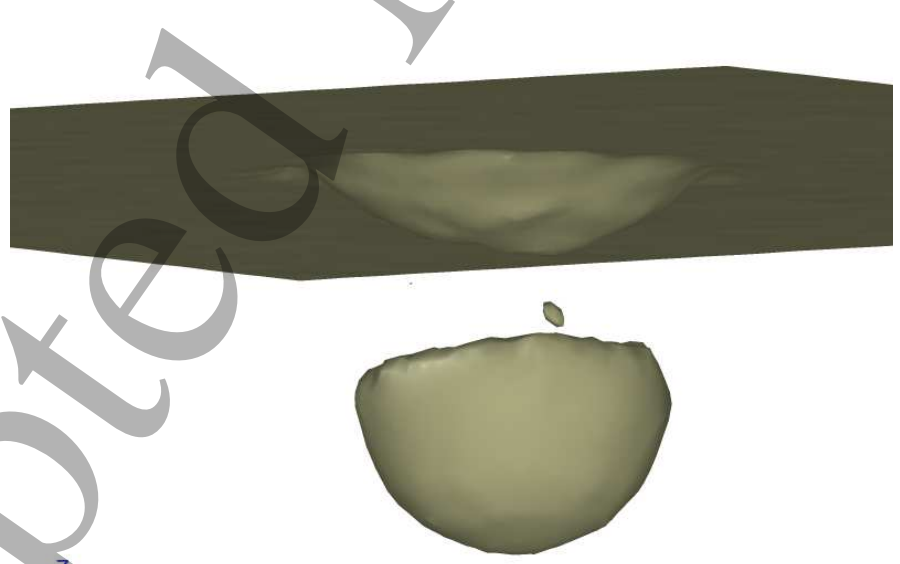

(c)

FIG. 4: Residual defective structures for [100]-oriented crystals with voids at different depths. a) $37 \AA$, b) $27 \AA$ and c) 14 A. Green lines correspond to $\boldsymbol{b}=1 / 2\langle 111\rangle$ dislocations, while magenta lines correspond to $\boldsymbol{b}=\langle 100\rangle$ dislocations. 


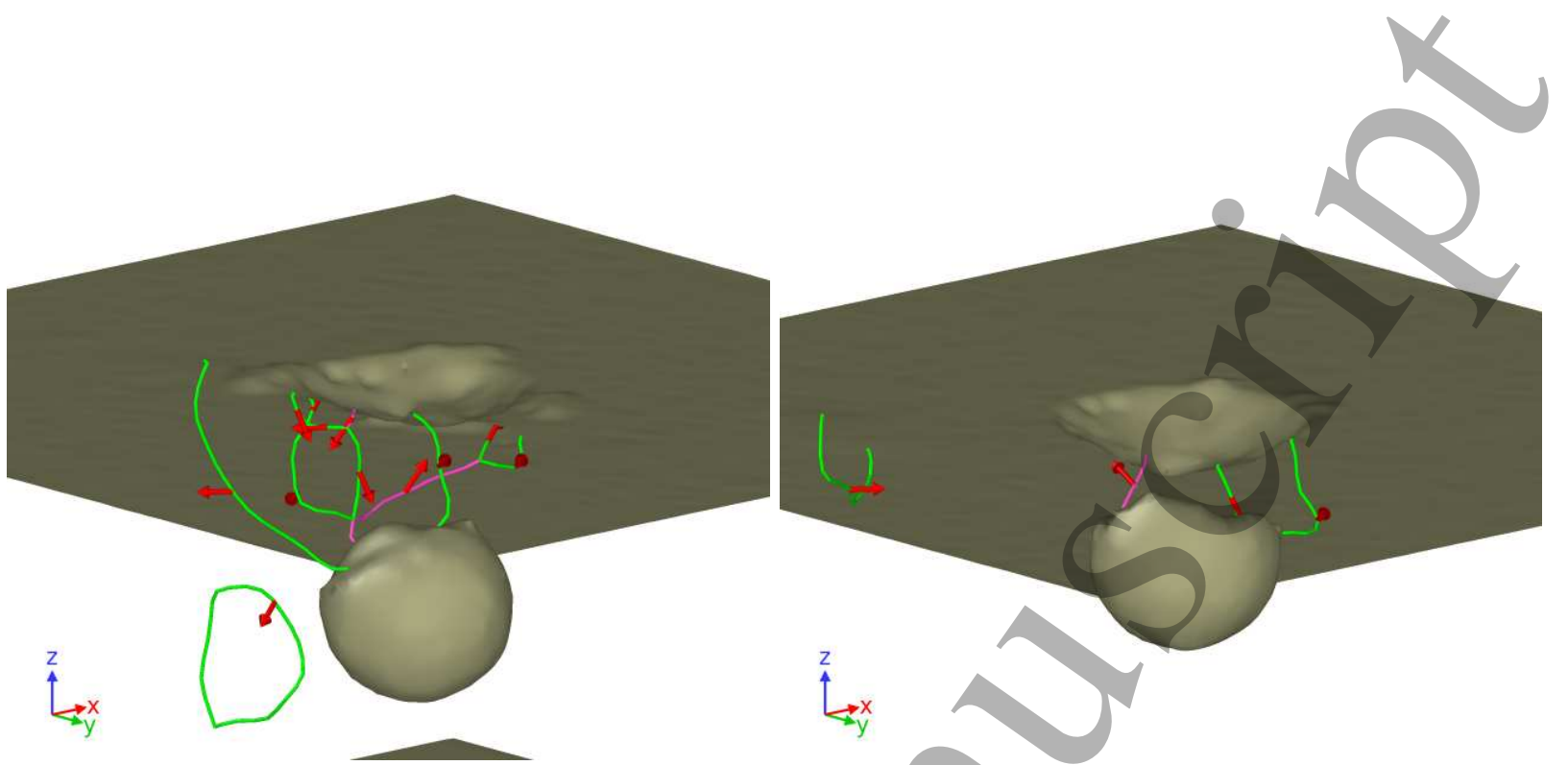

(b)

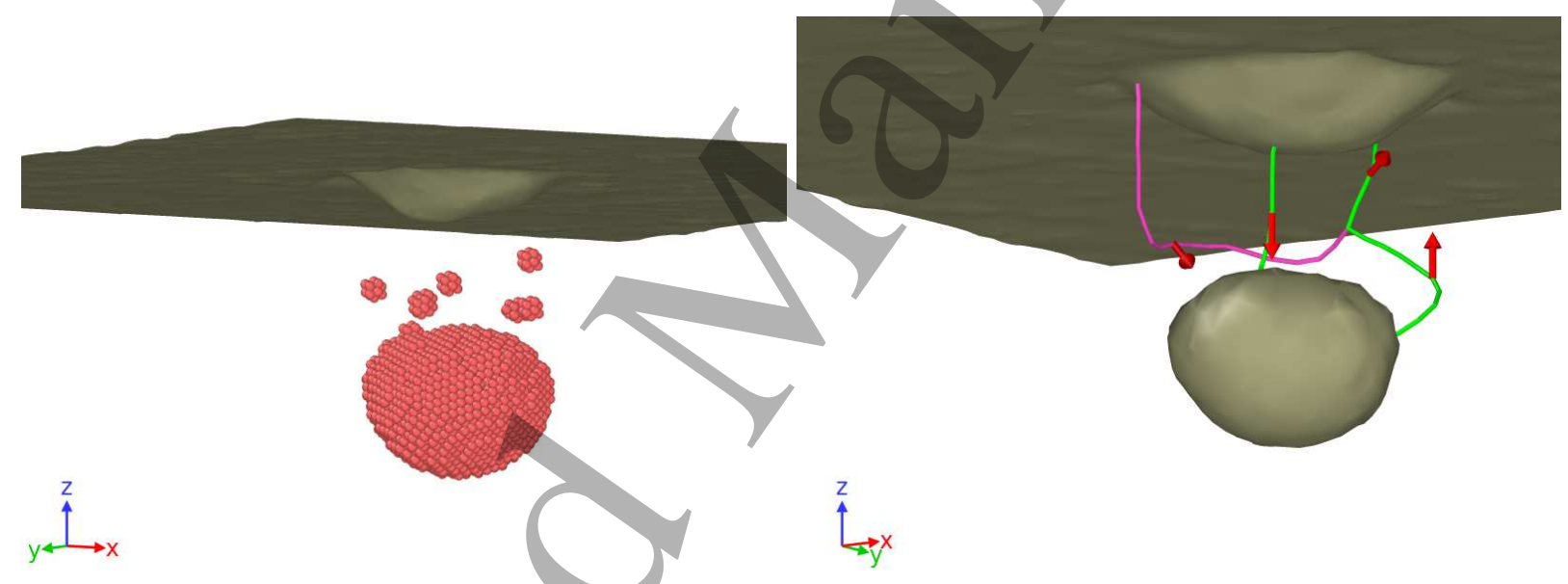

(c)

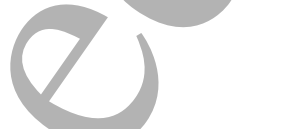

(d)

FIG. 5: Residual defective structures for [110] and [111] oriented crystals with voids at different depth. a) [110], $d=37 \AA, \mathrm{b})$ [110], $d=27 \AA$, c) [111], $d=37 \AA$, and d) [111], $d=27 \AA$. Green lines correspond to $\boldsymbol{b}=1 / 2\langle 111\rangle$ dislocations, while magenta lines correspond to $\boldsymbol{b}=\langle 100\rangle$ dislocations. Red arrows illustrate the Burgers vectors. 


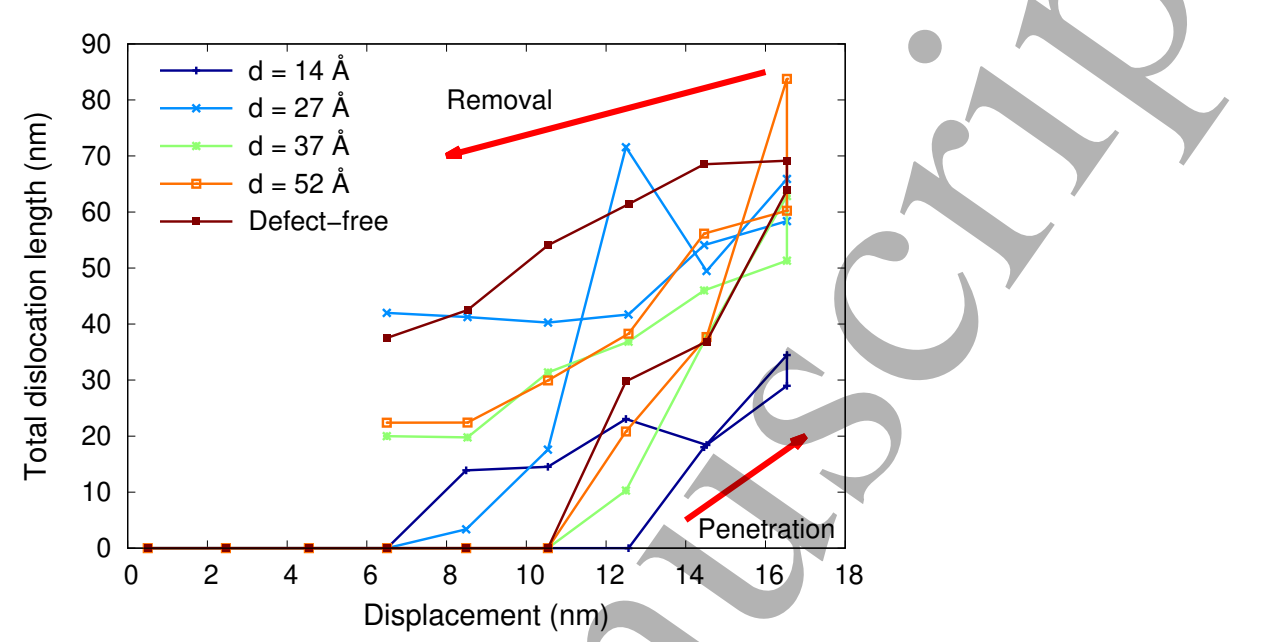

(a)

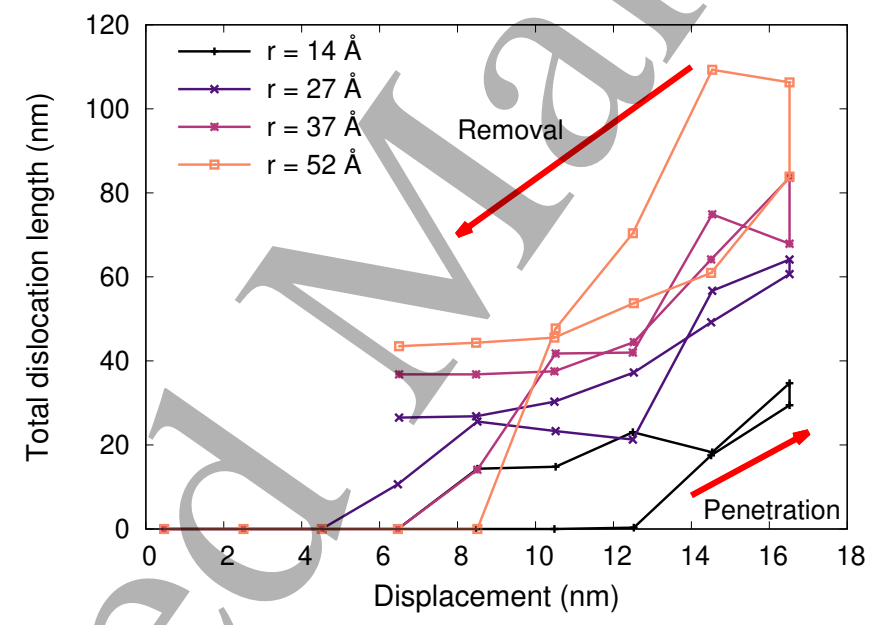

(b)

FIG. 6: Evolution of total dislocation length for a subset of simulations on [100] oriented substrates. a) Indentation with a 25 $\AA$ void and varying $d$. b) Indentation on samples with variable void radius and constant $d=14 \AA$. 

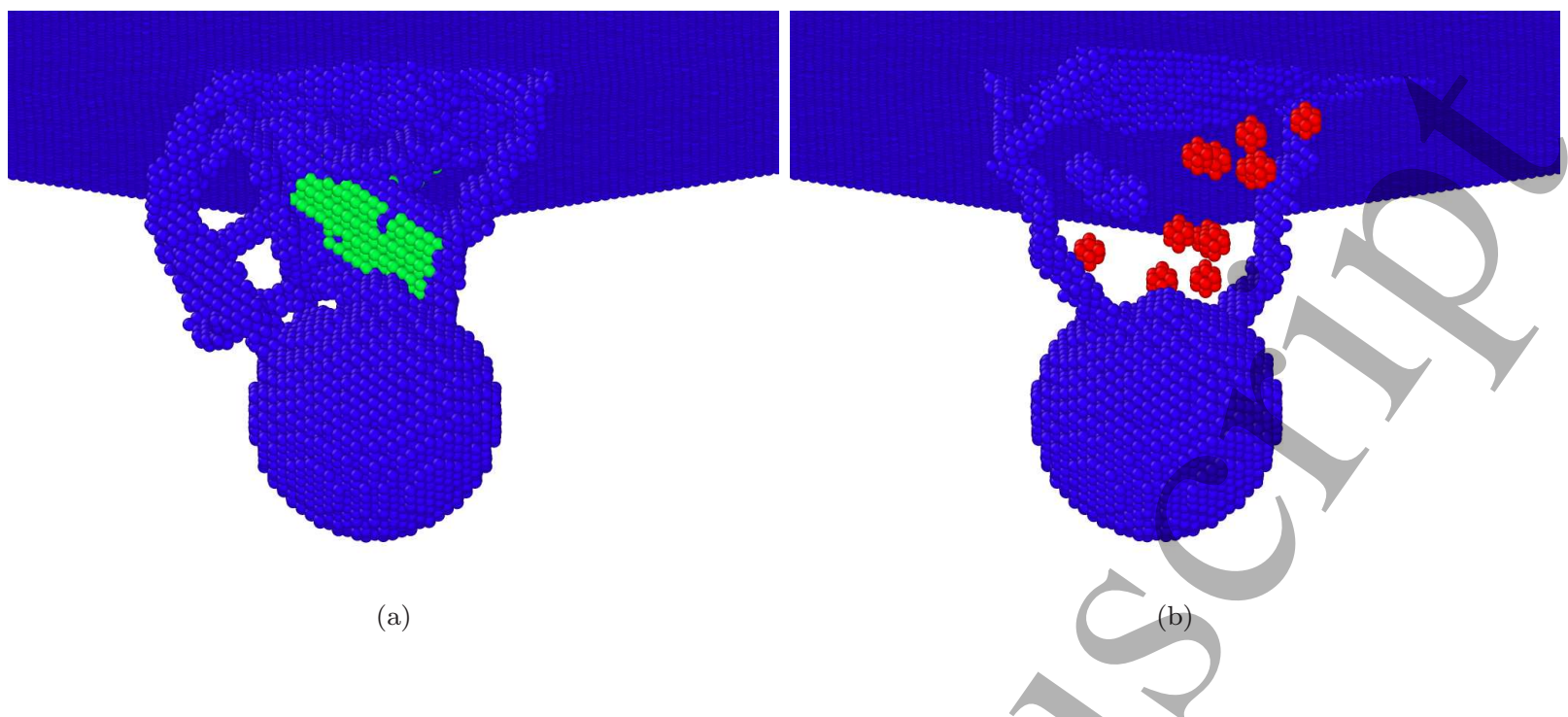

FIG. 7: Example of twinning found for a simulation on a [100] oriented substrate, with a $25 \AA$ void at depth $d=52 \AA$ at a) maximum penetration and b) after unloading. Blue atoms correspond to free surfaces and dislocation cores, green atoms correspond to twin boundaries, while red atoms correspond to single and di-vacancies. Identification performed by means of CAT [35-37].

(a)

FIG. 8: Effect of indenter size on plasticity for [100] oriented substrates containing a $25 \AA$ void at depth $d=14 \AA$. a) $R=25$ $\AA$, b) $R=50 \AA$ Green lines correspond to $\boldsymbol{b}=1 / 2\langle 111\rangle$ dislocations, while magenta lines correspond to $\boldsymbol{b}=\langle 100\rangle$ dislocations. 
a)

20

21

22

23

24

25

26

27

28

29

30

31

32

33

34

35

36

37

38

39

40

41

42

43

44

45

46

47

48

49

50

51

52

53

54

55

56

57

58

59

60
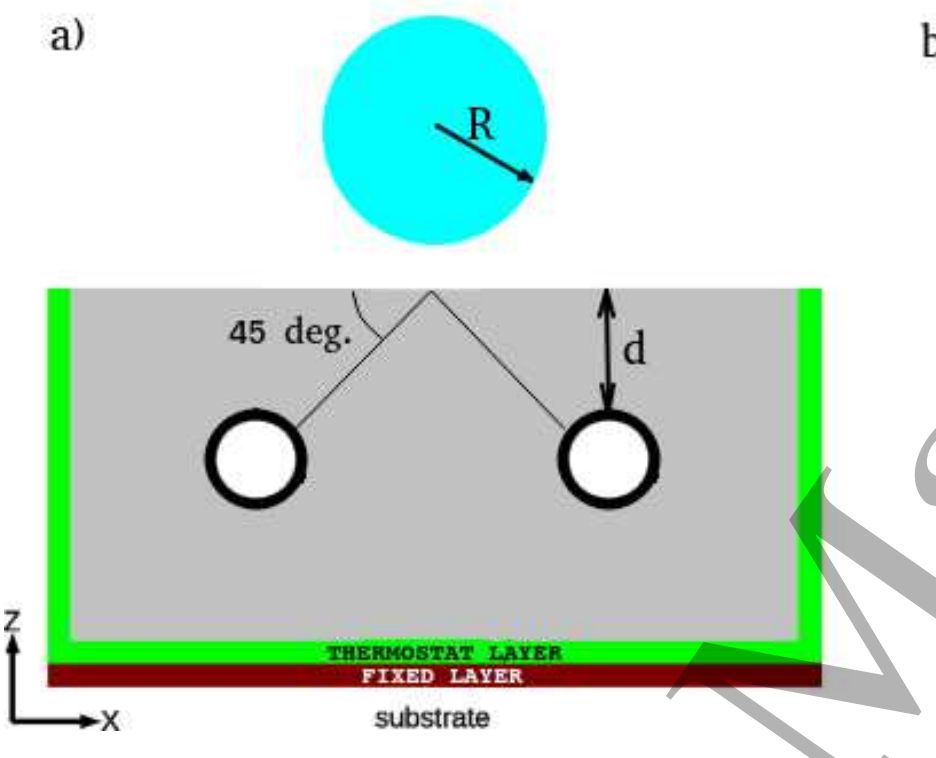

b)

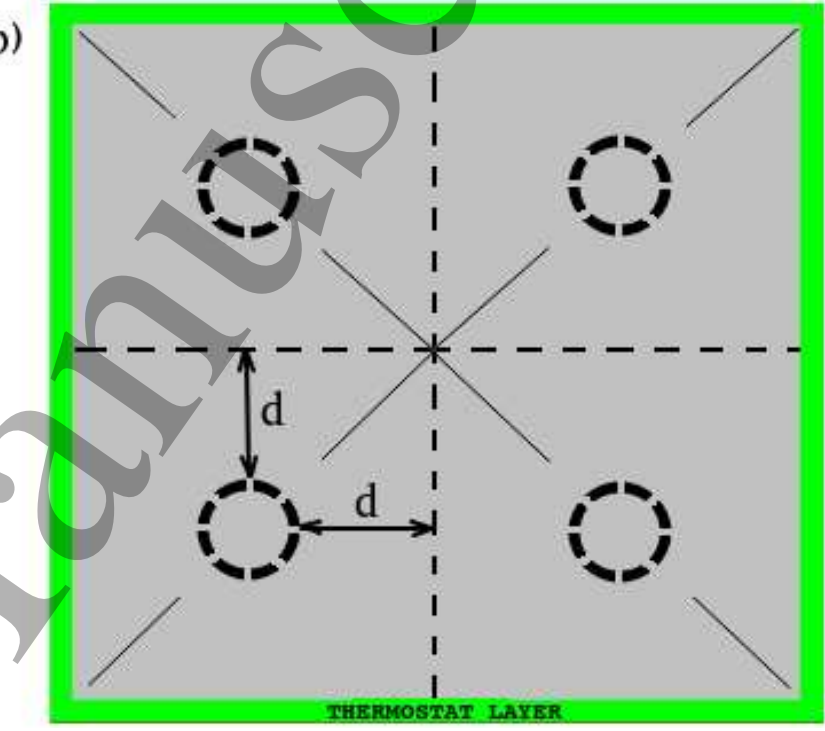

FIG. 9: Setup of the simulation system with 4 voids strategically oriented along the $\langle 111\rangle$ directions from the indenter contact point at the substrate surface. The radius $R$ of the indenter and the distance between the upper apex of each void and the surface $d$ are indicated. The substrate has thermostatting and rigid zones at its boundaries. 


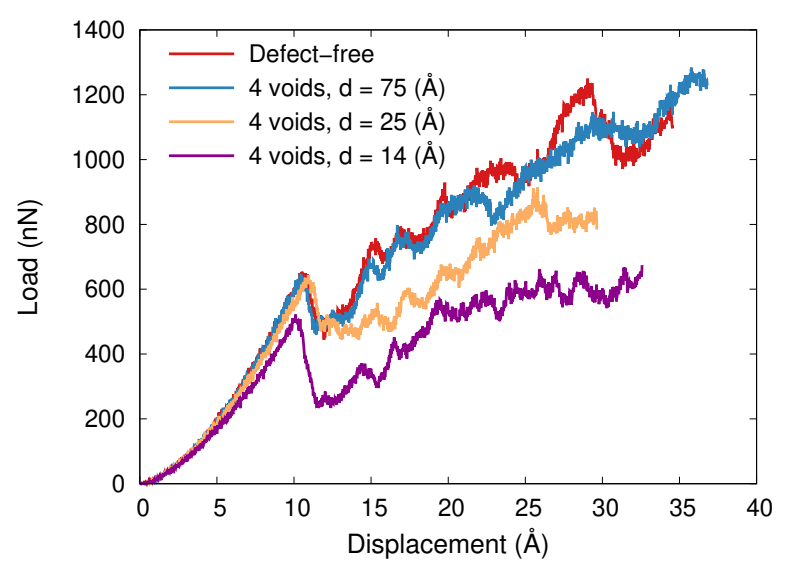

(a)

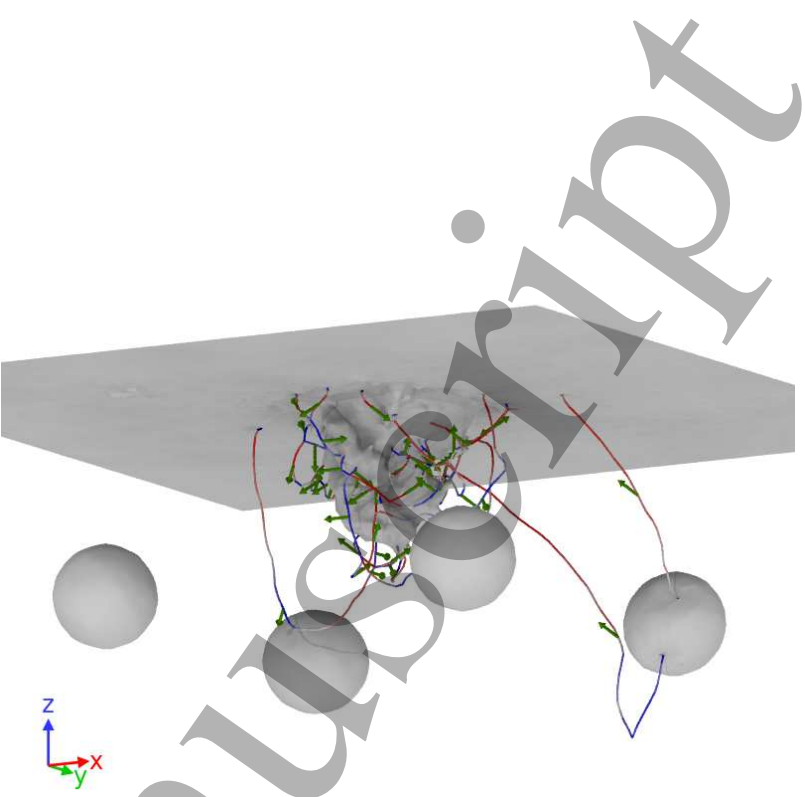

(b)

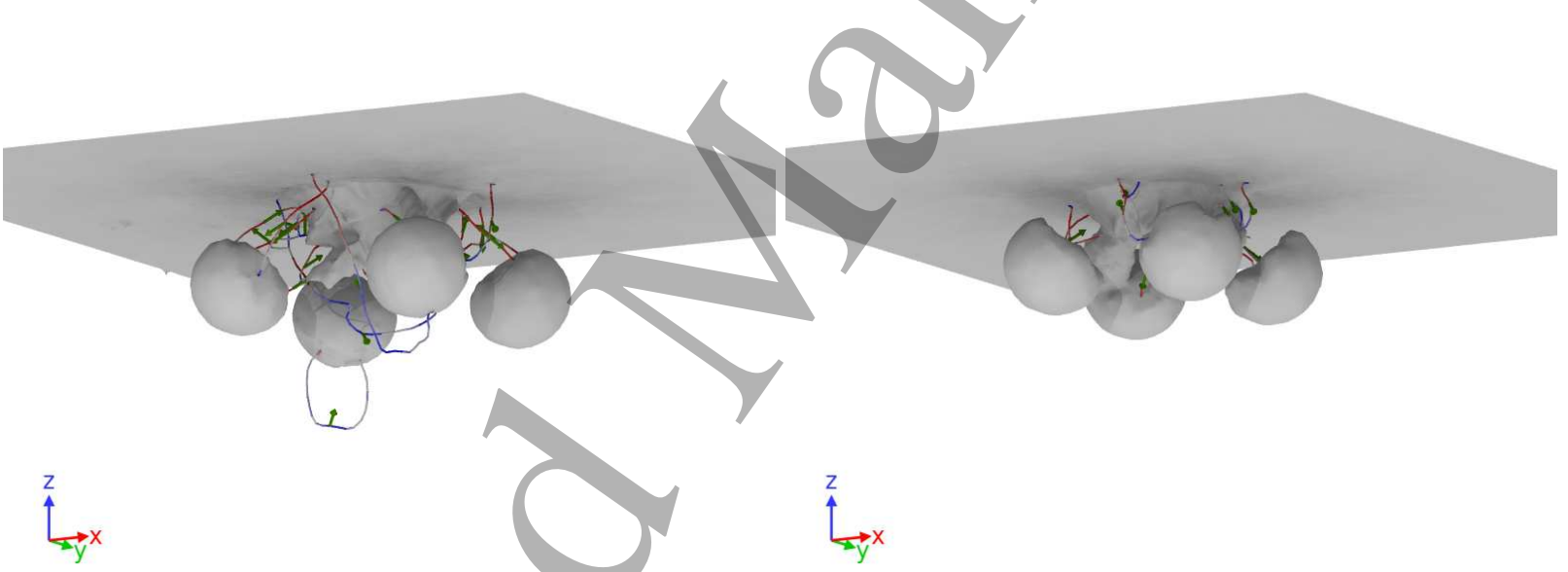

(c)

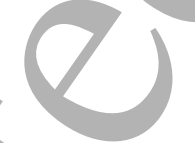

(d)

FIG. 10: a) Loading curves for a varietyj of pore positions. Defective structures for a displacement of $30 \AA$ corresponding samples with an array of voids at b) a depth of $75 \AA$, c) a depth of $27 \AA$, and d) a depth of $14 \AA$. 


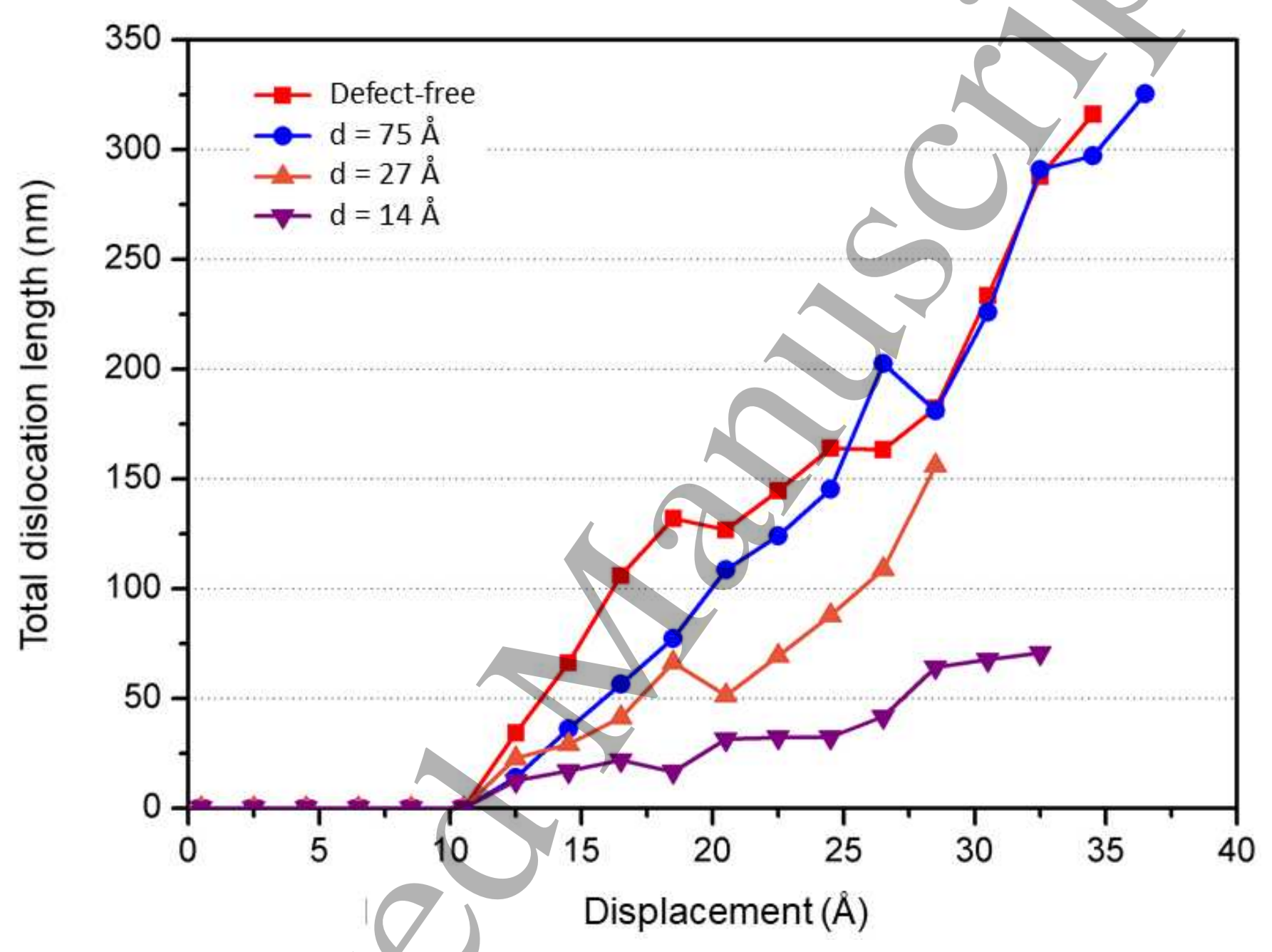

FIG. 11: Evolution of total dislocation length for indentation of an array of voids. 


\begin{tabular}{|c||c|c|c||c|c|c|}
\hline \multicolumn{1}{|c||}{} & \multicolumn{3}{c||}{ after indent } & \multicolumn{3}{c|}{ after removal } \\
\hline Case & $L_{\text {disl }}(\mathrm{nm})$ & $R_{\mathrm{pl}}(\mathrm{nm})$ & $\rho\left(10^{16} \mathrm{~m}^{-2}\right)$ & $L_{\text {disl }}(\mathrm{nm})$ & $R_{\mathrm{pl}}(\mathrm{nm})$ & $\rho\left(10^{16} \mathrm{~m}^{-2}\right)$ \\
\hline defect-free & 69.5 & 9.8 & 3.5 & 37.5 & 8.2 & 3.2 \\
\hline$d=52 \AA$ & 82 & 11.8 & 2.4 & 23 & 6.7 & 3.6 \\
\hline$d=37 \AA$ & 63 & 7.6 & 6.8 & 20 & 6.6 & 3.3 \\
\hline$d=27 \AA$ & 48 & 6.3 & 9.1 & 42 & 5.5 & 12 \\
\hline$d=14 \AA$ & 34.5 & 6.1 & 7.2 & 0 & N/A & N/A \\
\hline
\end{tabular}

TABLE I: Dislocation densities corresponding to the curves presented in Fig. 6 after full indentation and after indenter retraction.

$L_{\text {disl }}$ : total dislocation length within plastic zone,

$R_{\mathrm{pl}}$ : radius of plastic zone,

$\rho$ : dislocation density. 